\title{
Assessment of Myocardial Damage in Chronic Chagasic Patients using QRS Slopes
}

\author{
E Pueyo $^{1}$, E Laciar $^{2}$, E Anzuola $^{1}$, P Laguna $^{1}$, R Jané $^{3}$ \\ ${ }^{1}$ Instituto de Investigación en Ingeniería de Aragón (I3A), and CIBER-BBN, Univ. Zaragoza, Spain \\ ${ }^{2}$ Gabinete de Tecnología Médica, Universidad Nacional de San Juan, Argentina \\ ${ }^{3}$ Dept ESAII, CREB, Universitat Politècnica de Catalunya, Barcelona, Spain
}

\begin{abstract}
In this study the slopes of the QRS complex are evaluated for determination of the degree of myocardial damage in chronic chagasic patients. Previous studies have demonstrated the ability of the slope indices to reflect alterations in the conduction velocity of the cardiac impulse. Results obtained in the present study show that chronic chagasic patients have significantly flatter QRS slopes as compared to healthy subjects. Not only that but the extent of slope lessening turns out to be proportional to the degree of myocardial damage caused by the disease. Additionally, when incorporating the slope indices into a classification analysis together with other indices indicative of the presence of ventricular late potentials obtained from high resolution electrocardiography, results show that the percentages of correct classification increase up to $62.5 \%$, which means eight points above the percentages obtained prior to incorporation of the slope indices. It can be concluded that QRS slopes have great potential for assessing the degree of severity associated with Chagas' disease.
\end{abstract}

\section{Introduction}

Chagas' disease is an infectious illness caused by the parasite Tripanosoma cruzi, which is transmitted to humans trough the feces of a bug called Triatoma infestans. The World Health Organization (WHO) estimates that 16 to 18 million people in Latin American countries are already infected by the disease and other 100 million people are at risk of being infected [1].

In general terms, two different stages of Chagas' disease can be distinguished. The first stage, called acute phase, appears shortly after the parasitical infection and it is occasionally manifested by high temperature, inflammations, and heart rate acceleration. Following this phase, which lasts for 1 or 2 months, there is an undetermined latent period. After that, some patients go into a chronic phase, which is characterized by alterations in the cardiovascular system, normally associated to the so-called Chagas' cardiomyopathy. This type of cardiomyopathy produces malfunctioning in the propagation of the electrical impulse as well as destruction of cardiac fibers. In areas where the illness is endemic, Chagas' cardiomyopathy represents the first cause of cardiovascular death [2].

In order to optimize treatment for chronic chagasic patients, it is essential to make use of an effective diagnosis tool able to determine the existence of cardiac injury and, if positive, its magnitude. Clinical diagnosis is usually based on tests such as chest x-rays, echocardiogram, or electrocardiogram (ECG), which can be either Holter ECG or conventional rest ECG. The use of high-resolution electrocardiography (HRECG) has been reported in the literature as a useful tool for clinical assessment of Chagas' disease $[3,4]$. Specifically, the presence of ventricular late potentials (VLP) has been detected in chronic chagasic patients using high-resolution ECGs. VLP, which are usually measured on temporally averaged beats, are very lowamplitude high-frequency signals found within the terminal part of the QRS complex and the beginning of the ST segment.

Since Chagas' cardiomyopathy frequently leads to alterations in the heart's electrical conduction, in this study we propose the evaluation of QRS slopes for determination of the degree of myocardial damage associated with the disease. In the literature, the standard method used to identify conduction defects is the estimation of the QRS highfrequency content $(150-250 \mathrm{~Hz})$. However, it has been recently shown that QRS slopes represent more robust indices, with enhanced performance in situations where high-frequency QRS indices were traditionally used, such as for ischemia monitoring [5]. In the present study, we hypothesize that chagasic patients have less steep slopes as compared to healthy subjects, and the factor of lessening is directly related to the degree of myocardial injury. The ability of the proposed slope indices will be assessed in high-resolution recordings of individuals for which a classification is already available from a set of conventional 
clinical tests.

\section{Study population}

The study population is composed of 107 individuals from the Chagas database recorded at Simón Bolívar University (Venezuela) [4]. For each individual, a continuous 10-minute HRECG was recorded using orthogonal XYZ lead configuration. All the recordings were digitized with a sampling frequency of $1 \mathrm{kHz}$ and amplitude resolution of 16 bits.

Out of the total 107 individuals of the study population, 96 are patients with positive serology for Tripanosoma cruzi, classified in 3 groups according to the evolution of Chagas' cardiomyopathy (Groups I, II, and III). The other 11 individuals are healthy subjects with negative serology taken as a control group (Group 0). The four analyzed groups are described in detail next:

- Group 0: 11 healthy subjects, mean age $33.6 \pm 10.9$ years, 9 males, 2 females.

- Group I: 41 chagasic patients, mean age $41.4 \pm 8.1$ years, 21 males, 20 females. They show no evidence of myocardial damage in either echocardiogram, conventional 12-lead ECG or Holter ECG.

- Group II: 39 chagasic patients, mean age $45.8 \pm 8.8$ years, 19 males, 20 females. They present normal echocardiogram, but they show some evidence of weak or moderate myocardial damage in either conventional or Holter ECG.

- Group III: 16 chagasic patients, mean age $53.6 \pm 9.3$ years, 9 males, 7 females. They have reduced ejection fraction, severe myocardial damage in conventional ECG and premature ventricular contractions or episodes of ventricular tachycardia in Holter ECG.

\section{Methods}

\subsection{HRECG}

\subsubsection{QRS slopes}

In this work, evaluation of QRS slopes is performed for all of the HRECG recordings of the database described in Section 2. For each recording, let's denote $x_{i}(n)$, $n=0, \ldots, N$, the i-th beat of lead $\mathrm{X}$, where $\mathrm{i}$ runs froms 0 to I (being I the total number of beats in the recording). Analogously, let's denote $y_{i}(n)$ and $z_{i}(n)$ the ith beats of leads Y and Z, respectively. QRS slopes are measured on temporally averaged signals $\bar{x}(n), \bar{y}(n)$, and $\bar{z}(n), n=0, \ldots, N$, which are calculated as the average of all normal beats $i=0, \ldots, I$ of the recording. Ectopic and grossly noisy beats are excluded of the averaging process. The averaging is performed following the standard recommendations described in [6].
A three-step process is applied to compute the upward QRS slope, $\alpha_{\mathrm{US}}$, and the downward QRS slope, $\alpha_{\mathrm{DS}}$, of each averaged beat $\bar{x}(n), \bar{y}(n)$, and $\bar{z}(n)$. In the first step, delineation is performed using a wavelet-based technique [7] that determines the temporal locations of $\mathrm{Q}, \mathrm{R}$, and $\mathrm{S}$ wave peaks, which are denoted by $n_{\mathrm{Q}}, n_{\mathrm{R}}$, and $n_{\mathrm{S}}$, respectively [5]. The second step identifies the time instant $n_{\mathrm{U}}$ associated with maximum slope of the ECG signal (i.e., global maximum of its derivative) between $n_{\mathrm{Q}}$ and $n_{\mathrm{R}}$. Analogously, the time instant $n_{\mathrm{D}}$ corresponding to minimum slope of the ECG signal between $n_{\mathrm{R}}$ and $n_{\mathrm{S}}$ is identified. As a final step, a line is fitted in the least squares sense to the ECG signal in a window of $15 \mathrm{~ms}$ around $n_{\mathrm{U}}$, and the slope of that line is defined as $\alpha_{\mathrm{Us}}$. In the same manner, $\alpha_{\mathrm{DS}}$ is defined as the slope of a line fitted in a $15-\mathrm{ms}$ window around $n_{\mathrm{D}}$

\subsubsection{Ventricular late potentials}

Other temporal indices defined to detect the presence of VLP in HRECG recordings are also evaluated in this work. Previous studies in the literature have shown the ability of those indices to determine the severity of Chagas' cardiomyopathy [8]. Consequently, the purpose of our study is to investigate the benefits of using such indices in conjunction with the QRS slopes.

Computation of VLP indices considers filtered leads X, $\mathrm{Y}$, and Z using a bi-directional 4th-order Butterworth filter with passband between 40 and $250 \mathrm{~Hz}$. The filtered signals are denoted by $x_{i, f}(n), y_{i, f}(n)$,and $z_{i, f}(n)$. The indices QRSD, RMS40, and LAS40, which are described next, require temporal signal averaging (beats $\bar{x}_{f}(n), \bar{y}_{f}(n)$, and $\left.\bar{z}_{f}(n)\right)$ as well as calculation of the vector magnitude defined as: $v(n)=\sqrt{\bar{x}_{f}^{2}(n)+\bar{y}_{f}^{2}(n)+\bar{z}_{f}^{2}(n)}$.

On the signal $v(n)$ the three indices defined to detect VLP are computed based on identification of time instants $n_{\mathrm{b}}$ and $n_{\mathrm{e}}$ corresponding to beginning and end of the QRS complex [6]:

$$
\begin{aligned}
\mathrm{QRSD} & =n_{\mathrm{e}}-n_{\mathrm{b}} \\
\mathrm{RMS} 40 & =\left(\frac{1}{n_{2}-n_{1}} \sum_{n=n_{1}}^{n_{2}} v^{2}(n)\right)^{1 / 2},\left\{\begin{array}{l}
n_{1}=n_{\mathrm{e}}-40 \mathrm{~ms} \\
n_{2}=n_{\mathrm{e}}
\end{array}\right. \\
\mathrm{LAS} 40 & =n_{\mathrm{e}}-\operatorname{argmax}\{n \mid v(n) \geq 40 \mu V\} .
\end{aligned}
$$

\subsection{Clinical analysis}

The proposed QRS slope indices $\alpha_{\mathrm{US}}$ and $\alpha_{\mathrm{DS}}$ are compared between the groups of individuals described in Section 2, with the objective of determining whether the probability distributions of the four groups are significantly different. For the statistical comparison, the nonparametric Mann-Whitney U test is used. 
Table 1. P-values from Mann-Whitney $\mathrm{U}$ test in the comparison of groups using indices $\alpha_{\mathrm{US}}$ and $\alpha_{\mathrm{DS}}$.

\begin{tabular}{ccccccc}
\hline Index & 0 vs I & 0 vs II & 0 vs III & I vs II & 1 vs III & II vs III \\
\hline$\alpha_{\text {US }}$ & 0.130 & $<0.001$ & $<0.001$ & $<0.001$ & $<0.001$ & 0.124 \\
$\alpha_{\text {DS }}$ & 0.031 & $<0.001$ & $<0.001$ & 0.0015 & $<0.001$ & 0.385 \\
\hline
\end{tabular}

Additionally, a discriminant analysis is performed to assess the capability of the QRS slope indices for classification of chagasic patients in groups I to III. In that analysis, the indices $\alpha_{\mathrm{US}}, \alpha_{\mathrm{DS}}$, QRSD, RMS40, and LAS40 are considered as independent variables and their performance is evaluated grouping them in subsets of variable length running from 1 to 5 .

\section{4. $\quad$ Results}

\subsection{QRS slopes and myocardial damage}

Evaluation of the two QRS slopes, $\alpha_{\mathrm{US}}$ and $\alpha_{\mathrm{DS}}$, on signal averaged beats shows that their mean values are very different for the four analyzed groups of individuals. Out of the three recorded leads, the largest differences are found in lead Y.

Figure 1 shows two bar graphics with the values of $\alpha_{\text {US }}$ and $\alpha_{\mathrm{DS}}$ measured in lead Y. In each graphic mean \pm standard error of the mean, separately calculated for the four groups, is presented. It can be observed that both QRS slopes become flatter as the degree of myocardial damage increases.
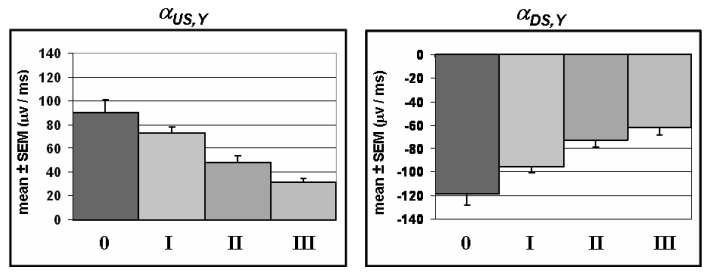

Figure 1. Mean and standard error of the mean (SEM) for the indices $\alpha_{\mathrm{US}}$ and $\alpha_{\mathrm{DS}}$ in each of the analyzed groups (0,I,II, and III), expressed in $\mu V / \mathrm{ms}$.

Application of the Mann-Whitney $U$ test for separation of group means leads to the p-values shown in Table 1 for $\alpha_{\mathrm{US}}$ and $\alpha_{\mathrm{DS}}$. According to those results, it can be concluded that, when comparing any of two groups of individuals, the distributions of QRS slope values turn out to be substantially different. The significance of the statistical tests is noticeably high in all cases, except when confronting groups 0 and I (where only $\alpha_{\mathrm{DS}}$ leads to significant results), and when confronting groups II and III (where neither $\alpha_{\mathrm{US}}$ nor $\alpha_{\mathrm{DS}}$ are significant).

Figure 2 presents an example of the above described results. Four averaged beats are shown, each one extracted from a HRECG recording of an individual of one of the four analyzed groups. The fitted lines used to compute
Table 2. P-values from Mann-Whitney U test in the comparison of groups using QRSD, RMS40, and LAS40.

\begin{tabular}{ccccccc}
\hline Index & 0 vs I & 0 vs II & 0 vs III & I vs II & 1 vs III & II vs III \\
\hline QRSD & 0.822 & 0.241 & $<0.001$ & 0.055 & $<0.001$ & 0.006 \\
LAS40 & 0.094 & 0.005 & $<0.001$ & 0.015 & $<0.001$ & 0.143 \\
RMS40 & 0.364 & 0.012 & $<0.001$ & 0.012 & $<0.001$ & 0.080 \\
\hline
\end{tabular}

$\alpha_{\mathrm{US}}$ and $\alpha_{\mathrm{DS}}$ are superimposed on each one of the averaged beats. It is apparent that both the upward and the downward strokes of the QRS complex are steeper for the healthy subject of Group 0 than for the chagasic patients. Moreover, the more the severity of Chagas' disease, the more noticeable become those differences.

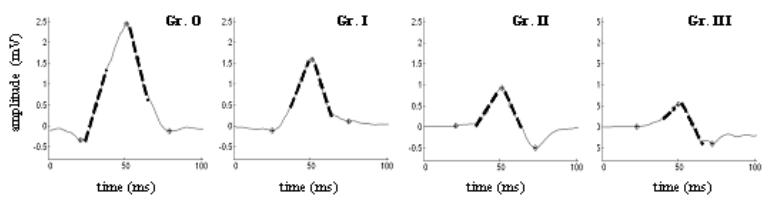

Figure 2. Examples of QRS slopes evaluation for individuals belonging to the 4 analyzed groups (0,I,II, and III)

\subsection{VLP and myocardial damage}

Figure 3 presents mean \pm standard error of the mean for the VLP indices described in Section 3.1.2. It can be appreciated that QRSD and LAS40 increase along with the extent of myocardial damage caused by Chagas disease, while RMS40 decreases. The p-values obtained from Mann-Whitney U test for separation of groups 0 , I, II, and III, using the VLP indices is shown in Table 2.
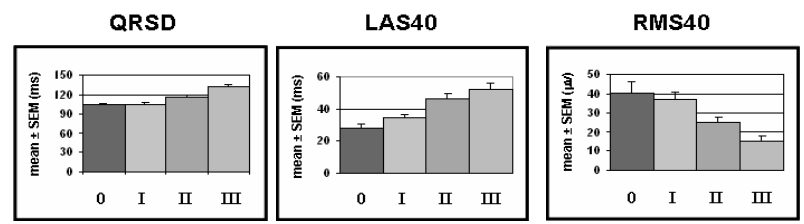

Figure 3. Mean and standard error of the mean (SEM) for the indices QRSD, RMS40, and LAS40 in each of the analyzed groups $(0, \mathrm{I}, \mathrm{II}$, and III), expressed in $\mathrm{ms}, \mathrm{ms}$ and $\mu V$, respectively.

\subsection{Classification of chagasic patients}

Since the proposed QRS slope indices, as well as the VLP indices, have shown to be significantly different, in mean, in the four analyzed groups, their use is considered for a classification analysis, with the purpose of identifying the progression of the disease in each patient.

Table 3 presents the results obtained from the classification analysis, using different combinations of indices. The percentages of correct classification for each of the three patient groups, as well as the total number of individuals 
Table 3. Percentages of correct classification derived from the discriminant analysis, using combinations of indices.

\begin{tabular}{ccccc}
\hline Combination of indices & \multicolumn{4}{c}{ Classification rate } \\
& Gr.I & Gr.II & Gr.III & Global \\
\hline RMS40 & 55.0 & 25.0 & 87.5 & 47.9 \\
$\alpha_{\text {US }}$ & 80.0 & 20.0 & 75.0 & 54.2 \\
$\alpha_{\text {DS }}$ & 60.0 & 15.0 & 87.5 & 45.8 \\
QRSD-LAS40 & 85.0 & 15.0 & 62.5 & 52.1 \\
QRSD- $\alpha_{\text {US }}$ & 80.0 & 60.0 & 42.5 & 60.4 \\
QRSD-LAS40-RMS40 & 80.0 & 20.0 & 62.5 & 52.1 \\
QRSD-RMS40- $\alpha_{\text {US }}$ & 80.0 & 45.0 & 62.5 & 62.5 \\
\hline
\end{tabular}

correctly classified, have been specified. From Table 3 it can observed that the consideration of the QRS slopes into the analysis improves the classification rates. In particular, when classification is performed by means of a unique variable, the best of the QRS-based indices for VLP detection (described in Section 3.1.2) leads to a global classification rate of $47.9 \%$, while $\alpha_{\text {US }}$ reaches a global rate of $54.2 \%$. When two variables are used in the analysis, the greatest classification percentage using indices from the set $\{$ QRSD, RMS40, LAS40 $\}$ is attained with the combination QRSD-LAS40 (52.1\%). The consideration of the index $\alpha_{\text {US }}$ together with any of the indices of that combination makes the classification percentage increase by around $8 \%$, reaching $60.4 \%$ with the pair of variables QRSD- $\alpha_{\text {US }}$. If groups of three variables are considered, the combination of $\alpha_{\text {Us }}$ with RMS40 and QRSD augments the correct classification rate up to $62.5 \%$. The inclusion of a higher number of variables in the discriminant analysis does not improve the attained classification percentage.

\section{Discussion and conclusions}

In this work the slopes of the QRS complex, $\alpha_{\mathrm{US}}$ and $\alpha_{\mathrm{DS}}$, have been evaluated on HRECG recordings of healthy subjects and chronic chagasic patients with different degrees of myocardial damage. The proposed indices have been compared with other indices defined to detect VLP [8].

Results described in Section 4.1 have corroborated our initial hypothesis, showing that both the upward and the downward slope of the QRS complex are less steep in chronic chagasic patients, and there is a close link between the extent of slope attenuation and the degree of cardiac injury caused by the disease. The physiological basis that supports the above described results could be related to the fact that most chronic chagasic patients present defects in the propagation of the cardiac electrical impulse. In [5] we provided evidence that QRS slopes are able to reflect delays in the conduction velocity, which could well explain the results found for patients affected by Chagas' disease.

Furthermore, the present study has shown that QRS slope indices $\left(\alpha_{\mathrm{US}}\right.$ and $\left.\alpha_{\mathrm{DS}}\right)$ represent an additional value for classification of chronic chagasic patients when they are taken together with other indices used for VLP detection (QRSD, RMS40, LAS40). As stated in Section 4.3, the combination of variables QRSD-RMS40- $\alpha_{\text {Us }}$ provides a percentage of correct classification of $62.5 \%$, which is $8 \%$ above the classification rate obtained before incorporating the slope indices into the analysis. The fact that the optimum combination for performing the classification includes the upward slope $\alpha_{\text {US }}$ in conjunction with the indices QRSD and RMS40 suggests that the information provided by the QRS slopes (in particular, $\alpha_{\mathrm{US}}$ ) is complementary to that provided by the other two QRS-based indices. Even though QRSD is to a certain extent related to the slopes, there are QRS changes that can be reflected by the slopes but not by QRSD, since definition of the slope indices gathers information from the amplitude as well as from the duration of the QRS complex. On the other hand, the index RMS40 is associated with the energy of the final portion of the QRS complex, which does not directly influence the evaluation of QRS slopes.

\section{Acknowledgements}

This work has been supported by Ministerio de Ciencia y Tecnología and FEDER through project TEC200405263-C02 and by DGA, Spain, through GTC ref:T30.

\section{References}

[1] Chagas Disease Elimination. Burden and Trends. WHO Division of Control of Tropical Diseases ;.

[2] Maguire JH, Hoff R, Sherlock I, Guimaraes AC, C. SA, B. RN, E. MK, H. ST. Cardiac morbidity and mortality due to chagas' disease: prospective electrocardiographic study of a brazilian community. Circulation 1987;75:1140-45.

[3] Dopico L, Nadal J, Infantosi A. Analysis of late potentials in the high-resolution electrocardiogram of patients with chagas' disease using weighted coherent average. Revista Brasileira de Engenharia Biomedica 2000;16(1):49-59.

[4] Mora F, Gomis P, Passariello G. Seales electrocardiográficas de alta resolución en chagas- El proyecto SEARCH. Acta Cientifica Venezolana 1999;50:187-194.

[5] Pueyo E, Sornmo L, Laguna P. QRS slopes for detection and characterization of myocardial ischemia. IEEE Trans Biomed Eng 2007; Preprint TBME-00803-2006.

[6] Breithardt G, Cain ME, El-Sherif N, Flowers NC, Hombach V, Janse M, B. SM, Steinbeck G. Standards for analysis of ventricular late potentials using high-resolution or signalaveraged electrocardiography. Circulation 1991;83.

[7] Martínez JP, Almeida R, Olmos S, Rocha AP, Laguna P. A wavelet-based ECG delineator: evaluation on standard databases. IEEE Trans Biomed Eng 2004;51.

[8] Laciar E, Jané R, Brooks DH, Torres A. Análisis de seal promediada y latido a latido del ECG de alta resolución en pacientes con mal de Chagas. In Actas del XXIV Congreso Anual de la Sociedad Espaola de Ingeniería Biomédica. Sociedad Espaola de Ingeniería Biomédica, 2006; 169-172. 